\title{
Recepción y uso de medios de comunicación en los jóvenes fronterizos
}

\author{
José Carlos Lozano \\ Instituto Tecnológico y de Estudios Superiores \\ de Monterrey (ITESM) - Campus Monterrey
}

OMO EN la gran mayoría de las áreas y temáticas relacionadas con la comunicación de masas en México, es muy poco lo que sabemos actualmente sobre los patrones concretos de exposición y uso de medios de comunicación en diferentes regiones del país y en los diferentes grupos sociales que componen la audiencia. Con demasiada frecuencia, encontramos en el estudio de la comunicación en México un impresionante aparato conceptual sobre los fenómenos y procesos de la comunicación masiva, correspondido por una dramática escasez de investigaciones empíricas. Como han señalado Fuentes y Sánchez Ruiz (1992), gran parte de la investigación en esta área se caracteriza por ser generalista y centralizada, concentrándose en "la generación de teoría que 'lo explique todo' de una sola vez" y minimizando el trạajo empírico (p. 17). En otra obra, Sánchez Ruiz (1993: 40) afirma...

hemos tendido a ser más filosofos, poetas y periodistas que científicos sociales. Nuestros grandes rollos y discursos teóricos y políticos, o nuestras descripciones coyunturales, no han bastado para desentrafar, explicar y comprender satisfactoriamente la realidad comunicativa y social latinoamericana.

Las razones de este problema son diversas. Van desde el teoricismo heredado de los setenta (véase Prieto 1984) hasta la deficiente capacitación metodológica de muchos investigadores y la carencia de recursos económicos y humanos.

Lo anterior explica el por qué es tan escasa la bibliografía disponible sobre la recepción de mensajes comunicacionales en México. Mientras que en otros países hace décadas que investigan directamente los efectos de la televisión y los demás 
medios en las audiencias, en nuestro país - y en América Latina en general - no hemos podido en cierta medida ni reunir los datos descriptivos más elementales. Esfuerzos como los de Cremoux (1968), Rotta (s. d.), Fernández Collado (1986), Montoya y Rebeil (1986), Sánchez Ruiz (1989), González (1988) y Áceves (1991), entre otros, ${ }^{1}$ han sido muy valiosos y han aportado información útil para conocer los patrones de exposición y uso de la televisión, pero son muy pocos y ofrecen panorámicas limitadas en lo espacial (cubren sólo algunas ciudades o regiones del país), en lo temporal (fueron realizadas en diferentes años, lo que impide su integración), y en los medios analizados (por lo general sólo la televisión), por no hablar de las diferencias en indicadores y aproximaciones conceptuales y metodologicas.

De esta forma, estamos en un nivel donde necesitamos, además de investigaciones que se concentren en detectar procesos de apropiación, negociación y refuncionalización de contenidos - según sugieren las teorías culturalistas actuales - estudios que aporten datos básicos sobre patrones de exposición, uso y percepción de los medios por parte de los receptores. Se requiere sistematizar la información existente por edades, clases sociales, sexo, regiones y demás variables socio-demográficas. Se requiere, asimismo, replicar en otros lugares y actualizar las pocas investigaciones disponibles, saber qué grupos sociales, en qué momentos y con qué intensidad se exponen a qué medios y a qué contenidos.

\section{La Investigación sobre el consumo de medios en la Frontera Norte}

$\mathrm{Al}$ igual que sobre otras regiones del país, la investigación relativa a patrones de exposición y uso de medios en la frontera norte ha sido escasa y esporádica. Pese a que las entidades federativas de esta región ocupan los primeros lugares nacionales en número de periódicos y estaciones radiofónicas y televisivas (véase Lozano, 1991a), y que numerosos investigadores consideran que la exposición a medios de comunicación estadounidenses ha propiciado un debilitamiento de la identidad nacional, ${ }^{2}$ son casi inexistentes los estudios de recepción de medios.

1 A estos esfuerzos aislados habría que agregar los estudios recientes sobre el consumo de televisión y periódicos en el valle de México publicados por la revista Internedios (1992, Núms. 2 y 3).

2 Para una discusión sobre los autores que han afirmado lo anterior véase Lozano (1991b). 
Entre los pocos trabajos que incluyen datos sobre la exposición a mensajes comunicacionales en esta parte del país se encuentra el de Bustamante (1983), donde entre muchas otras variables no relacionadas con los medios se presentan datos sobre exposición a la prensa y la televisión. Según esta encuesta, aplicada en Tijuana, Baja California, Ciudad Juárez, Chihuahua, Matamoros, Tamaulipas, y la Ciudad de México, los jóvenes fronterizos leían el periódico diariamente en mayor proporción que sus contrapartes capitalinas (pp. 50-52), y su exposición a la televisión estadounidense se correlacionaba con la clase social: los jóvenes de clase alta (de las tres ciudades fronterizas y de la Ciudad de México) veían más programas televisivos estadounidenses que los de clase media y baja (p. 59). Otro de los estudios más difundidos es el de Malagamba (1986) sobre la exposición de los niños de primaria de Tijuana a la televisión de ambos países. Con una muestra de 300 niños de cuarto año de primaria, la investigadora de El Colegio de la Frontera Norte encontró que la población infantil de ese nivel mostraba una mayor preferencia por programas originados y producidos en México que por los estadounidenses (p. 32).

El presente trabajo busca contribuir al conocimiento de los patrones de consumo de medios de comunicación en el país en general y en la frontera norte en particular mediante la aplicación de una encuesta por muestreo a estudiantes de secundaria de Nuevo Laredo. Aligual que las investigaciones previasya reportadas, este trabajo tiene claras limitaciones espaciales y temporales. Se concentra en una sola ciudad de la región, y en un sólo segmento poblacional, lo que dificulta su generalización más allá de esos dos aspectos. Asimismo, por su naturaleza transversal, refleja los patrones de consumo y uso de medios prevalecientes únicamente en el momento de la encuesta y las entrevistas (noviembre-diciembre de 1991), no la evolución de las preferencias ni la situación actual. Sin embargo, al igual que los estudios previos, el presente ofrece datos puntuales sobre los hábitos de exposición de este grupo de jóvenes a la radio, la televisión y el cine que pueden ser útiles como un primer acercamiento a la problemática y que pueden sumarse a las evidencias previas en el resto del país.

Las preguntas de investigación se relacionaron con la obtención de datos básicos sobre la disponibilidad, acceso y hábitos de exposición a los medios. 
1) ¿Qué disponibilidad tienen los alumnos de secundaria de Nuevo Laredo de las diferentes tecnologías de comunicación masiva (radio, televisión, videocasetera, cable, antena parabólica)?

2) ¿Con qué frecuencia se exponen a la radio, la televisión y el cine?

En un segundo nivel, se plantearon interrogantes sobre el posible impacto de los medios y contenidos estadounidenses en la identidad cultural de los jóvenes fronterizos. Como hemos argumentado en otro momento (Lozano, 1993), para que los contenidos estadounidenses ejerzan un impacto determinado en la identidad cultural de las audiencias mexicanas se requiere el cumplimiento de las siguientes condiciones: a) Que haya disponibilidad de medios y contenidos extranjeros en los sistemas de comunicación nacionales; b) que las audiencias se expongan a ellos significativamente; c) que la exposición a dichos contenidos redunde en la asimilación de los valores ideológicos extranjeros por parte de las audiencias; y d) que esa asimilación propicie realmente un debilitamiento o pérdida de identidad cultural. Para responder al segundo aspecto (el primero se encuentra implícito en la descripción de la oferta de medios y contenidos de ambos lados de la frontera en Nuevo Laredo), este trabajo planteó las siguientes preguntas adicionales:

3) ¿Existe unà exposición masiva a los medios de comunicación estadounidenses o a mensajes estadounidenses disponibles en los medios mexicanos por parte de los jóvenes de secundaria de Nuevo Laredo?

4) ¿Existen algunas diferencias en la exposición a medios y contenidos nacionales y estadounidenses por clase social, sexo, años de residencia en la frontera y conocimiento del idioma inglés?

\section{Método}

El trabajo de campo se realizó en Nuevo Laredo, Tamaulipas, ciudad situada junto a la frontera de México con el estado estadounidense de Texas. Con una población cercana a los 250 mil habitantes, Nuevo Laredo es una de las principales ciudades de la frontera norte de México. Constituye el principal puerto terrestre mexicano para el cruce de importaciones y exportaciones; la economía gravita casi en su totalidad en dicho intercambio, facilitado por numerosos funcionarios públicos, agencias aduanales, compañías de transporte de carga y múltiples servicios indi- 
rectos. En este sentido, se manifiesta una intensa vinculación económica con la ciudad vecina (Laredo, Texas) y con Estados Unidos en lo general.

Con respecto a la disponibilidad de medios de comunicación, Nuevo Laredo cuenta con una gran diversidad de los mismos, tanto locales y nacionales ${ }^{3}$ como extranjeros. En la ciudad existían en 1991 dos estaciones de televisión (una local con películas mexicanas y estadounidenses y series de acción estadounidenses dobladas al español y la otra repetidora de la señal del Canal 2 de Televisa), 12 radiodifusoras, cinco salas cinematográficas y alrededor de 50 establecimientos de renta de videos nacionales y extranjeros. De Laredo, Texas se recibían sin interferencia alguna en los aparatos receptores las señales de 3 canales de televisión (uno afiliado a la cadena NBC, otro a la CBS y el tercero a la cadena hispana Telemundo, con programación en español) y 5 radiodifusoras. Los neolaredenses podían cruzar al lado estadounidense y asistir a alguna de las 7 salas cinematográficas, y rentar o comprar películas en video en numerosos establecimientos destinados a ese fin. En un contexto de gran gama de opciones comunicacionales, el análisis de posibles consumos diversificados se tornaba factible.

La encuesta se planteó como objetivo diagnosticar los patrones generales de exposición a la radio, la televisión y el cine (incluyendo el uso de videocasetera) por parte de los jóvenes de secundaria de Nuevo Laredo y explorar algunas relaciones básicas entre variables específicas como clase social y uso de medios.

El presente estudio se realizó a fines de 1991 en Nuevo Laredo, Tamaulipas, y consistió en 575 encuestas y 30 entrevistas en profundidad con jóvenes de las escuelas secundarias de dicha ciudad. En total, contestaron la encuesta 263 hombres y 300 mujeres, 161 alumnos de clase alta, 295 de clase media y 107 de clase baja. El diseño muestral fue por etapas. Primero se obtuvo el permiso de la inspección local de secundarias de la SEP para realizar la encuesta; dicha dependencia proporcionó el listado de las escuelas existentes y el número de alumnos en cada

3 Nuevo Laredo, como la mayor parte de las ciudades ubicadas en los estados mexicanos que colindan con los Estados Unidos, cuenta con un número mayor de medios locales y nacionales que sus contrapartes del centro o sur del país. Véase (Iglesias, 1991; Toussaint, 1991; Lozano, 1991). Según Lozano (1991), mient ras que las entidades norteñas tenían en 1990 el 16\% de la población nacional, contaban con el $28 \%$ del total de periódicos, el $32 \%$ de las estaciones de radio y el $29 \%$ de las estaciones de televisión del país. Esto, explicado por el mayor dinamișmo económico de esta región del país debido a su cercanía con los Estados Uniđos, sugiere que si bien los fronterizos pueden recibir señales televisivas y radiofónicas norteamericanas en sus receptores, también cuentan con una oferta muy amplia de medios locales y nacionales. 
una de ellas por grado y sexo. El siguiente paso fue clasificar las escuelas por nivel socio-económico de los alumnos. ${ }^{4}$ En noviembre de 1991, había 8,486 alumnos de secundaria en la ciudad. De esos, 8,070 asistían a escuelas públicas y 403 a las privadas. Para asegurar un número adecuado de casos de clase alta, se decidió sobremuestrear las escuelas privadas en razón de 7 encuestas en ellas por cada 1 de las aplicadas en las públicas. Así, el número final de la muestra fue 563 y no las 425 que le correspondían al universo de 8,486 alumnos con un nivel de confianza de $95 \%$ y un error de estimación del $5 \% .^{5}$ A continuación se determinó el número de encuestas que le correspondían a cada secundaria pública o privada existente en Nuevo Laredo, y ya dentro de cada una de ellas a cada grado, de acuerdo con su población estudiantil. Las direcciones de las escuelas proporcionaron las listas del total de alumnos de cada grado, las cuales sirvieron como base de la muestra para seleccionar al azar el número de alumnos correspondiente a su peso en el universo. El cuestionario fue auto-aplicado en salones en que se juntó al total de alumnos de cada escuela previamente seleccionados.

El cuestionario fue piloteado con 30 estudiantes de secundaria, 10 de cada estrato social ( 5 hombres y 5 mujeres).

\section{Resultados}

\section{Patrones de recepción de medios}

En total, 563 estudiantes contestaron el cuestionario. El Cuadro 1 muestra que 263 eran hombres y 300 mujeres; 161 eran de clase alta (incluyendo el sobremuestreo de este segmento), 295 de clase media y 107 de clase baja.

El Cuadro 2 muestra los patrones de disponibilidad de aparatos de radio y televisión, videocaseteras, televisión por cable y antenas parabólicas. Casi todos

4 Este, sin embargo, no fue el único determinante de clase social. En el cuestionario se incluyeron variables referentes a características de la vivienda, ocupación de los padres y medio de transporte de estos últimos, que en conjunto con la clasificación original de la escuela, permitieron ubicar la clase social de cada estudiante muestreado.

5 En el análisis de los datos se manejan los dos diferentes totales, 425 o 563, dependiendo de las inferencias deseadas. En cuadros que se refieren a los alumnos de secundaria en general se usa el total de 425 encuestas (para regresar a su justa proporción en el universo a los alumnos de clase alta). En cuadros que estratifican a los alumnos por clase social, se usa el total de 563 , para permitir un mayor número de casos en la clase alta. 
Cuadro 1. Número de alumnos de secundaria encuestados por sector socio-económico y sexo

(Diciembre 1991)

\begin{tabular}{crrr}
\hline SEcTOR & HOMBRES & MUJERES & TOTAL \\
Sector I & 72 & 89 & 161 \\
Sector II & 139 & 156 & 295 \\
Sector III & 52 & 55 & 107 \\
& & & \\
Total & 263 & 300 & 563 \\
\hline
\end{tabular}

Cuadro 2. Tenencia de aparatos de televisión, radio, videocasetera, cable y antena parabólica en los hogares de los alumnos, por sector socioeconómico

\begin{tabular}{|c|c|c|c|c|}
\hline TENENCIA & SEGTORI & SECTOR II & SECTOR III & TOTAL \\
\hline \multicolumn{5}{|l|}{ Televisión } \\
\hline Si & 100 & 99.7 & 94.3 & 98.8 \\
\hline No & - & 0.3 & 5.7 & 1.2 \\
\hline Total & $\begin{array}{c}100 \% \\
(n=161)\end{array}$ & $\begin{array}{c}100 \% \\
(n=299)\end{array}$ & $\begin{array}{c}100 \% \\
(n=106)\end{array}$ & $\begin{array}{c}100 \% \\
(n=566)\end{array}$ \\
\hline \multicolumn{5}{|l|}{ Radio } \\
\hline SI & 100 & 99.0 & 99.1 & 99.3 \\
\hline No & - & 1.0 & 0.9 & 0.7 \\
\hline Total & $\begin{array}{c}100 \% \\
(n=158)\end{array}$ & $\begin{array}{c}100 \% \\
(n=297)\end{array}$ & $\begin{array}{l}100 \% \\
(n=106)\end{array}$ & $\begin{array}{c}100 \% \\
(n=561)\end{array}$ \\
\hline \multicolumn{5}{|c|}{ Videocasetera } \\
\hline Si & 96.3 & 68.0 & 47.1 & 72.3 \\
\hline No & 3.8 & 32.0 & 52.9 & 27.7 \\
\hline Total & $\begin{array}{c}100 \% \\
(n=160)\end{array}$ & $\begin{array}{c}100 \% \\
(n=297)\end{array}$ & $\begin{array}{c}100 \% \\
(n=102)\end{array}$ & $\begin{array}{c}100 \% \\
(n=559)\end{array}$ \\
\hline \multirow{2}{*}{\multicolumn{5}{|c|}{$\frac{X 2=80.949, \text { g.l. } 2, p_{1}=.0000}{\text { Cable }}$}} \\
\hline & & & & \\
\hline si & 40.3 & 13.5 & 6.3 & 19.8 \\
\hline No & 59.7 & 86.5 & 93.8 & 80.2 \\
\hline Total & $\begin{array}{c}100 \% \\
(n=149)\end{array}$ & $\begin{array}{c}100 \% \\
(n=281)\end{array}$ & $\begin{array}{l}100 \% \\
(n=96)\end{array}$ & $\begin{array}{c}100 \% \\
(n=526)\end{array}$ \\
\hline \multirow{2}{*}{\multicolumn{5}{|c|}{$\begin{array}{l}X 2=57.44, \text { g.l. } 2, p .=.0000 \\
\text { Antena parabólica }\end{array}$}} \\
\hline \multicolumn{2}{|c|}{ Antena parabólica } & & & \\
\hline SI & 47.7 & 4.9 & 2.0 & 16.2 \\
\hline No & 52.3 & 95.1 & 98.0 & 83.8 \\
\hline Total & $\begin{array}{c}100 \% \\
(n=149)\end{array}$ & $\begin{array}{c}100 \% \\
(n=287)\end{array}$ & $\begin{array}{c}100 \% \\
(n=100)\end{array}$ & $\begin{array}{c}100 \% \\
(n=536)\end{array}$ \\
\hline \multicolumn{5}{|c|}{$X 2=57.44$, g.1. $2, p_{1}=.0000$} \\
\hline
\end{tabular}


los estudiantes tenían en sus casas, por lo menos, un aparato de televisión y un radio. La disponibilidad de videocaseteras, cable y antenas, por el contrario, mostraba una distribución más en consonancia con la clase social. Mientras que el $96.3 \%$ de los alumnos de clase alta tenían videocasetera, sólo el $68 \%$ de la clase media y el $\mathbf{4 7 . 1 \%}$ de la clase baja contaban con ella. La televisión por cable no se ha distribuido ampliamente en la ciudad debido a limitaciones técnicas y de capital de la compañía local, pero aún así el $\mathbf{4 0 . 3 \%}$ de los alumnos de clase alta tenían dicho servicio, en contraste con sólo el $13.5 \%$ de los de clase media y el $6.3 \%$ de los de clase baja. De hecho; los estudiantes de clase alta tenían mayor acceso a programación televisiva por antena parabólica que por sistema de cable; el $47.7 \%$ tenían este medio en casa, porcentaje radicalmente diferente al de sus contrapartes de clase media (4.9\%) y baja (2\%). En suma, todos los estudiantes, sin distinción de clase social, tendían a tener acceso a los medios tradicionales - television y radio - , mientras que el acceso a las nuevas tecnologías se mostraba diferenciado por la posición socio-económica. ${ }^{6}$

\section{Exposición a diferentes medios}

Muchos estudios sobre el impacto de los medios de comunicación en la audiencia tienden a enfocar su atención en la televisión, argumentando que es por mucho el medio que las audiencias seleccionan más frecuentemente. Aunque esto parece ser cierto, no significa que otros medios como la radio, el cine, la prensa y las revistas sean marginales para las audiencias. De hecho, cotidianamente la gente usa otros medios además de la televisión, lo que podría mediar, reforzar o incluso diluir el impacto televisivo (véase Fuenzalida, 1989). El Cuadro 3 muestra que la exposición a la radio y el cine (tanto en salas públicas como en la videocasetera) no era tan amplia como para la televisión, pero sí importante. Los alumnos veían latelevisión un promedio de 242 minutos (4horas) diarias, mientras que escuchaban radio 164 minutos al día ( 2 horas con 44 minutos). Los estudiantes de clase baja eran los que dedicaban más tiempo a ambos medios, lo que demuestra que tenían menos opciones de entretenimiento que sus compañeros de clase media y alta.

6 Es interesante observar que una de esas nuevas tecnologías, la videocasetera, se encontraba ya ampliamente disponible para las clases media y baja, debido principalmente a su bajo costo en comparación con las otras tecnologías. 
Cuadro 3. Frecuencia con que ven TV, escuchan la radio 0 ven películas en el cine o con la videocasetera, por sector y sexo

\begin{tabular}{|c|c|c|c|c|c|}
\hline SECTOR & $f$ & MinUtos & DESV.EST. & $F / t$ & SIGNIFIC. \\
\hline \multicolumn{6}{|l|}{ Ven televisión } \\
\hline Sector 1 & 161 & 248.75 & 118.03 & $F=3.33$ & \multirow[t]{3}{*}{$0.0366 \mathrm{a}$} \\
\hline Sector 11 & 299 & 230.43 & 117.57 & & \\
\hline Sector III & 104 & 264.45 & 141.54 & \multirow{5}{*}{$t=0.95$} & \\
\hline \multicolumn{4}{|c|}{ a No hay diferencia significativa entre los sectores 2 y $3^{*}$} & & \multirow{4}{*}{0.343} \\
\hline Hombres & 262 & 247.68 & 134.48 & & \\
\hline Mujeres & 298 & 237.67 & 112.29 & & \\
\hline Global & 567 & 241.93 & 122.96 & & \\
\hline \multicolumn{6}{|l|}{ Escuchan radio } \\
\hline Sector 1 & 139 & 131.79 & 100.58 & \multirow[t]{3}{*}{$F=12.64$} & \multirow[t]{3}{*}{$0.0000 \mathrm{a}$} \\
\hline Sector II & 282 & 162.94 & 119.63 & & \\
\hline Sector III & 101 & 213.06 & 159.38 & & \\
\hline \multicolumn{6}{|c|}{ a No hay diferencia significativa entre los tres sectores } \\
\hline Hombres & 238 & 152.79 & 122.19 & \multirow{3}{*}{$t=-1.94$} & \multirow[t]{3}{*}{0.052} \\
\hline Mujeres & 281 & 174.34 & 130.04 & & \\
\hline Global & 567 & 164.34 & 126.57 & & \\
\hline \multicolumn{4}{|c|}{ Días por semana que ven películas con la videocasetera } & \multirow{4}{*}{$F=4.40$} & \multirow{4}{*}{$0.0130 a$} \\
\hline Sector 1 & 121 & 3.14 & 1.92 & & \\
\hline Sector II & 172 & 2.71 & 1.74 & & \\
\hline Sector III & 42 & 2.26 & 1.40 & & \\
\hline \multicolumn{4}{|c|}{ a Sólo hay diferencia significativa entre los sectores 1 y 3} & \multirow{4}{*}{$t=0.90$} & \multirow{4}{*}{0.371} \\
\hline Hombres & 147 & 2.72 & 1.75 & & \\
\hline Mujeres & 186 & 2.90 & 1.82 & & \\
\hline Global & 567 & 2.81 & 1.79 & & \\
\hline Van al cine & Sector I & Sector II & Sector III & \multirow{2}{*}{$\begin{array}{c}\text { Total } \\
19.5\end{array}$} & \\
\hline Una vez por semana & 13.9 & 19.1 & 28.8 & & \\
\hline Una vez por quincena & 13.9 & 10.1 & 8.7 & 10.9 & \\
\hline Una vez al mes & 13.9 & 10.7 & 12.5 & 12.0 & \\
\hline Ocasionalmente & 53.2 & 46.6 & 31.7 . & \multirow{2}{*}{$\begin{array}{l}45.7 \\
12.0\end{array}$} & \\
\hline Nunca & 5.1 & 13.4 & 18.3 & & \\
\hline \multirow[t]{2}{*}{ Total } & $\begin{array}{c}100 \% \\
(n=158)\end{array}$ & $\begin{array}{c}100 \% \\
(n=298)\end{array}$ & $\begin{array}{c}100 \% \\
(n=104)\end{array}$ & \multirow{2}{*}{$\begin{array}{c}100 \% \\
(n=560)\end{array}$} & \\
\hline & Hombres & Mujeres & Total & & \\
\hline Una vez por semana & 25.8 & 14.2 & 19.6 & & \\
\hline Una vez a la quincena & 12.7 & 9.5 & 11.0 & & \\
\hline Una vez al mes & 13.1 & 10.8 & 11.9 & & \\
\hline Ocasionalmente & 40.0 & 51.0 & 45.9 & & \\
\hline Nunca & 8.5 & 14.5 & 14.5 & \multirow{2}{*}{\multicolumn{2}{|c|}{$\begin{array}{c}\text { Procedimiento } \\
\text { b de Tukey }\end{array}$}} \\
\hline Total & $\begin{array}{c}100 \% \\
(n=260)\end{array}$ & $\begin{array}{c}100 \% \\
(n=296)\end{array}$ & $\begin{array}{c}100 \% \\
(n=556)\end{array}$ & & \\
\hline
\end{tabular}


En promedio, los estudiantes con videocasetera veían películas en ese medio tres veces a la semana (2.81). En este caso, los jóvenes de clase alta (3.14 días por semana) usaban esta tecnología con mayor intensidad que los de clase baja. Los últimos, sin embargo, asistían más frecuentemente al cine que los primeros. Casi el 30\% de los alumnos de clase baja asistían a salas cinematográficas una vez a la semana, contra sólo el $13.9 \%$ de los de clase alta.

\section{Exposición a medios estadounidenses}

Desde hace varios años, algunos académicos han externado temores en relación con la existencia de una masiva exposición de las audiencias fronterizas a los medios de comunicación estadounidenses o a sus mensajes disponibles en los medios locales y nacionales.

Los Cuadros 4 y 5 contestan la pregunta iqué tanto se exponen los jóvenes de secundaria de Nuevo Laredo a los medios estadounidenses que se reciben directamente en sus aparatos de radio y televisión? A pesar de poder sintonizar por lo menos dos canales televisivos estadounidenses (afiliados a las cadenas CBS y NBC) establecidos en Laredo, Texas, los estudiantes preferían con mucho las estaciones con programación mexicana o latinoamericana. El Cuadro 4 muestra que XHBR Canal 11 (Repetidor de la XEW Canal 2 de Televisa) era el preferido de los jóvenes, con una media de 4.41 en una escala de 1 a 5 (desde "nunca" hasta "todos los días"). Este resultado muestra que la proximidad geográfica a los Estados Unidos no parece propiciar cambios en la preferencia televisiva, ya que concuerda con la preferencia por el canal 2 de Televisa detectado por investigaciones realizadas en el interior del país (véase Aceves, 1991; García Canclini, 1993; González, 1988; Montoya y Rebeil, 1986).

La segunda estación más vista era la KLDo Canal $27($ media $=4.00$ ), que aunque establecida en el lado estadounidense, era repetidora de la cadena hispana Telemundo, con programación mexicana o latinoamericana. En tercer lugar de preferencia aparecía la televisora local XEFE Canal 2 con una media de 2.86 ("rara vez"), cuya programación se basa en telenovelas, películas mexicanas antiguas y algunas series de acción y películas dobladas al español. En los últimos dos lugares (del total de estaciones de recepción libre en Nuevo Laredo) se encontraban las dos televisoras de Laredo, Texas afiliadas a la CBS (media $=2.44$ ) y la NBC (media 2.07). 
Cuadro 4. Estaciones de televisión preferidas por los estudiantes, en recepción directa, cable y antena parabólica

Estaciones - MEDIA * DESV. ESTÁNDAR

En la televisión

(recepción directa)

XHBR (Televisa)

$\begin{array}{ll}4.41 & 0.88\end{array}$

KLDO (Telemundo)

$4.00 \quad 1.06$

XEFE (Local)

$2.86 \quad 0.99$

KVTV (CBS)

2.44

3.78

KGNS (NBC)

2.07

0.95

\section{En el cable}

Canales mexicanos

Canales de Estados Unidos

En la antena parabólica

Canales mexicanos

Canales de Estados Unidos

TOTAL

\subsection{4}

3.11
$1.31 n=61$

$1.26 n=62$

\footnotetext{
"Media en la escala 1 "Nunca", 2 "Rara vez", 3 "De vez en cuando", 4 "Con frecuencia" y 5 "Todos los días".
}

Los alumnos de clase media y baja suscritos al sistema de cable, a pesar de tener un mayor número de canales estadounidenses con programación en inglés, seguían prefiriendo con mayor frecuencia las estaciones mexicanas. En esto eran similares a los suscriptores del cable de León, Guanajuato, quienes preferían con bastante margen los canales mexicanos (XEW Canal 2, Galavisión, Canal 13) o de producción latinoamericana (Univisión y Telemundo) a pesar de tener acceso a varios estadounidenses (Revista Telemundo, 1992, p. 21). En contraste, los alumnos de clase alta, cuando utilizaban el cable, tendían a ver cadenas estadounidenses como HBO, Movie Channel, Pay Per View y otras, más que las mexicanas.

En suma, los estudiantes de secundaria, en general, tendían a ver canales mexicanos o con programación mexicana y latinoamericana con mucha mayor 
frecuencia que canales estadounidenses, excepto por un pequeño número de jóvenes de clase alta que sintonizaba canales estadounidenses en la parabólica.

La misma situación se repetía para las estaciones radiofónicas. El Cuadro 5 muestra que los estudiantes preferían abrumadoramente radiodifusoras locales con programación en español. Las 12 estaciones más escuchadas eran mexicanas, con un rango de frecuencia de escucha de 3.19 a 1.29 en la escala de 1 "nunca la escucho" a 5 "la escucho todos los días". Las cuatro estaciones ubicadas en Laredo, Texas estaban entre los últimos cinco lugares de preferencia de los alumnos.

Cuadro 5. Estaciones de radio preferidas por los alumnos

\begin{tabular}{|c|c|c|c|c|}
\hline RANGo & ESTACIÓN & TIPO DE MÚSICA & MEDIA & DESV. EST. \\
\hline 10. & XHTLN & Norteña & 3.19 & 1.36 \\
\hline 20. & XHNOE & Variada $\theta$ instrumental & 3.17 & 1.53 \\
\hline 30. & XHMW & Romántica & 2.96 & 1.29 \\
\hline 40. & XHNK & Moderna en español & 2.76 & 1.44 \\
\hline 50. & XEFE & Variada en español & 2.39 & 1.23 \\
\hline 60. & XEWL & Regional & 2.11 & 1.31 \\
\hline 70. & XENLT & Variada en español & 2.06 & 0.97 \\
\hline 80. & XEGNK & Ranchera & 1.75 & 1.06 \\
\hline 90. & XEAS & Tropical & 1.69 & 1.03 \\
\hline 100. & XEBK & Norteña & 1.56 & 0.87 \\
\hline 110. & XENU & Ranchera & 1.52 & 0.86 \\
\hline 120. & XEK & Variada & 1.39 & 0.73 \\
\hline 130. & KNOZ2"** & Contemporánea en inglés & 1.37 & 0.88 \\
\hline 140. & XEMU & Variada & 1.29 & 0.75 \\
\hline 150. & KOYE & Contemporánea en inglés & 1.23 & 0.69 \\
\hline 160. & $K L A R$ & Variada en español $\theta$ inglés & 1.22 & 0.66 \\
\hline 170. & KRRG & Variada en inglés & 1.21 & 0.66 \\
\hline
\end{tabular}

"Media en la escala: 1 "Nunca", 2 "Rara vez", 3 "De vez en cuando", 4 "Con frecuencia", 5 "Todos los días".

${ }^{\star \star}$ Las estaciones radiodifusoras con siglas en itálicas son estadounidenses que trasmiten desde Laredo, Texas. Todas comienzan con "K".

De esta forma, los jóvenes de secundaria de Nuevo Laredo, a pesar de su proximidad geográfica con los Estados Unidos yla posibilidad de sintonizar medios electrónicos estadounidenses, mostraban claros patrones de exposición y preferencia por los medios nacionales. 


\section{Exposición a contenidos estadounidenses}

Saber a qué medios de comunicación se exponían los alumnos era importante, pero no suficiente. La disponibilidad de programas estadounidenses de televisión en los canales mexicanos, de música en inglés en las radiodifusoras locales y de películas estadounidenses en los videoclubes y salas cinematográficas podía propiciar que se diera una exposición importante a dichos contenidos.

Programas televisivos. Los estudiantes de secundaria se veían claramente más interesados en programas producidos en México que en sus contrapartes estadounidenses. El Cuadro 6 muestra que de los 33 programas televisivos que obtuvieron más menciones, los primeros 16 habían sido producidos en México por Televisa, o en Estados Unidos, pero por Telemundo, con artistas latinos y en español. Siete de los primeros ocho programas más populares entre los alumnos eran producciones de Televisa como Papá soltero, Anabel, Chespirito, y telenovelas como Muchachitas. Hasta el décimo séptimo lugar aparecía el primer programa producido en Estados Unidos: caricaturas. Series estadounidenses de acción como Magnum, Duques de Hazzard y Chips aparecían en los últimos lugares, a pesar de estar dobladas al español y ser transmitidas por el canal local.

Así, la exposición de los jóvenes fronterizos a mensajes televisivos estadounidenses no era significativa, a pesar de vivir en la frontera con los Estados Unidos y tener distintas posibilidades para exponerse a ellos. De hecho, los estudiantes fronterizos tendían a ver menos programas estadounidenses que los niños de Guadalajara, los que según una encuesta de Sánchez Ruiz (1989: 61) tenían entre 10 sus programas favoritos a 7 procedentes de Estados Unidos).

Programación radiofónica y preferencias musicales. El Cuadro 7 muestra que la música moderna en español era la escuchada con mayor frecuencia en la radio, con una media de 3.87 en la escala de 1 ("nunca") a 5 ("todos los días"). En segundo lugar se encontraba la música popular mexicana (cumbias, norteñas, rancheras) con una media de 3.14, seguida de cerca por la música moderna en inglés (3.13).

La lista de los grupos o cantantes preferidos por los jóvenes demuestra que los artistas mexicanos eran mucho más populares que los estadounidenses. El Cuadro 8 muestra que el primer grupo estadounidense con cierta popularidad era Vanilla Ice, el cual ocupaba el octavo lugar de la lista, con 35 menciones, mientras que la vocalista Alejandra Guzmán (música moderna en español) y el Grupo Bronco (música norteña, grupera) se hallaban en primer lugar con 93 menciones. 
Cuadro 6. Programas de television preferidos por los alumnos

\begin{tabular}{|c|c|c|}
\hline RANGo & Programas & NÚM. MENCIONES ${ }^{\star}$ \\
\hline 10. & Telenovelas mexicanas (Televisa o XEFE) & 226 \\
\hline 20. & Papá soltero (Televisa) & 153 \\
\hline 20. & Anabel (Televisa) & 153 \\
\hline 40. & Chespirito (Televisa) & 144 \\
\hline 50. & Cine millonario (Telemundo) & 135 \\
\hline 60. & Cándido Pérez (Televisa) & 131 \\
\hline 70. & Muchachitas (Televisa) & 109 \\
\hline 80. & Ándale (Televisa) & 79 \\
\hline 90. & Cara a cara (Telemundo) & 64 \\
\hline 100. & Lucha libre mexicana (Televisa) & 51 \\
\hline 110. & Todo de todo (Televisa) & 45 \\
\hline 120. & Mujer, casos de la vida real (Televisa) & 40 \\
\hline 130. & wo (Televisa) & 35 \\
\hline 140. & Películas mexicanas (Televisa) & 34 \\
\hline 150. & Ocurrió así (Telemundo) & 33 \\
\hline 160. & La movida (Televisa) & 31 \\
\hline 160. & Caricaturas en inglés (NBC o cBS) & 31 \\
\hline 180. & A la cama con Pourcel (Telemundo) & 26 \\
\hline 190. & Caricaturas dobladas (XEFE) & 17 \\
\hline 200. & MTV International (Telemundo) & 16 \\
\hline 200. & Siempre en domingo (Televisa) & 16 \\
\hline 220. & Películas en Inglés (NBC O CBS) & 15 \\
\hline 230. & Primera tanda (Telemundo) & 13 \\
\hline 230. & Un millón de amigos (Telemundo) & 13 \\
\hline 250. & Magnum (XEFE, doblado) & 12 \\
\hline 250. & Cine en su casa (Telemundo) & 12 \\
\hline 270. & Contacto (Telemundo) & 11 \\
\hline 270. & Dukes of Hazzard (XEFE) & 11 \\
\hline 290 . & La telaraña (Televisa) & 10 \\
\hline 290. & Eco (Televisa) & 10 \\
\hline 290. & Chips (XEFE, doblado) & 10 \\
\hline \multirow[t]{6}{*}{290.} & Ninja Turtles (CBS) & 10 \\
\hline & Otros de Telemundo & 62 \\
\hline & Otros de Televisa o XEFE ${ }^{* *}$ & 59 \\
\hline & Otros en inglés & 53 \\
\hline & Otros de EU, doblados al español & 44 \\
\hline & $\begin{array}{l}\text { Otros mexicanos o latinoamericanos } \\
\qquad n=1,943\end{array}$ & 19 \\
\hline
\end{tabular}

* Cada alumno mencionó cinco de sus programas favoritos el total reportado es el de todas las menciones.

"Hay dos programas con menos de diez menciones, cada uno. 
Cuadro 7. Frecuencia con que ven los programas de televisión enlistados

\begin{tabular}{lcc}
\hline Programas & MEDiA & DESV. EsT. \\
\hline Papá soltero & 3.72 & 1.272 \\
Películas mexicanas & 3.72 & 0.992 \\
Anabel & 3.59 & 1.287 \\
Chespirito & 3.56 & 1.296 \\
Cándido Pérez & 3.51 & 1.273 \\
Películas de EU & 3.33 & 1.221 \\
Lucha libre mexicana & 2.98 & 1.678 \\
Tortugas ninja & 2.30 & 1.350 \\
Chips & 1.96 & 1.178 \\
Magnum & 1.84 & 1.173 \\
Daktari & 1.63 & 1.107 \\
Dukes of Hazzard & 1.57 & 1.003 \\
\hline
\end{tabular}

"Media en la escala: 1 "Nunca", 2 "Rara vez", 3 "De vez en cuando", 4 "Con frecuencia", 5 "Cada semana".

Al agrupar al total de grupos o vocalistas mencionados por los alumnos por el tipo de música que interpretan, se puede señalar que el $50.9 \%$ de las menciones fueron para los que interpretaban música moderna en español, $36.9 \%$ para los de música popular norteña y sólo el $12.2 \%$ para artistas estadounidenses (ver parte final del Cuadro 8). Estos resultados confirman los del Cuadro 9, que muestran la música en inglés en tercer lugar.

Cuadro 8. Frecuencia con que escuchan música en inglés, en español y ranchera

\begin{tabular}{lcc}
\hline Música & MEDIA $^{*}$ & DESV. EsT. \\
\hline Música en inglés & 3.35 & 1.275 \\
Música en español & 3.91 & 1.226 \\
Música ranchera & 2.89 & 1.375 \\
\hline
\end{tabular}

\footnotetext{
"Media en la escala: 1 "Nunca", 2 "Rara vez", 3 "A veces", 4 "Frecuentemente", 5 "Todos los días".
}

Películas preferidas en cine y video. Si los estudiantes preferían claramente la programación televisiva y la música mexicanas sobre sus contrapartes estadounidenses, se daba lo contrario en el caso de las películas. El Cuadro 9 muestra que 
Cuadro 9. Grupos musicales favoritos de los alumnos de secundaria

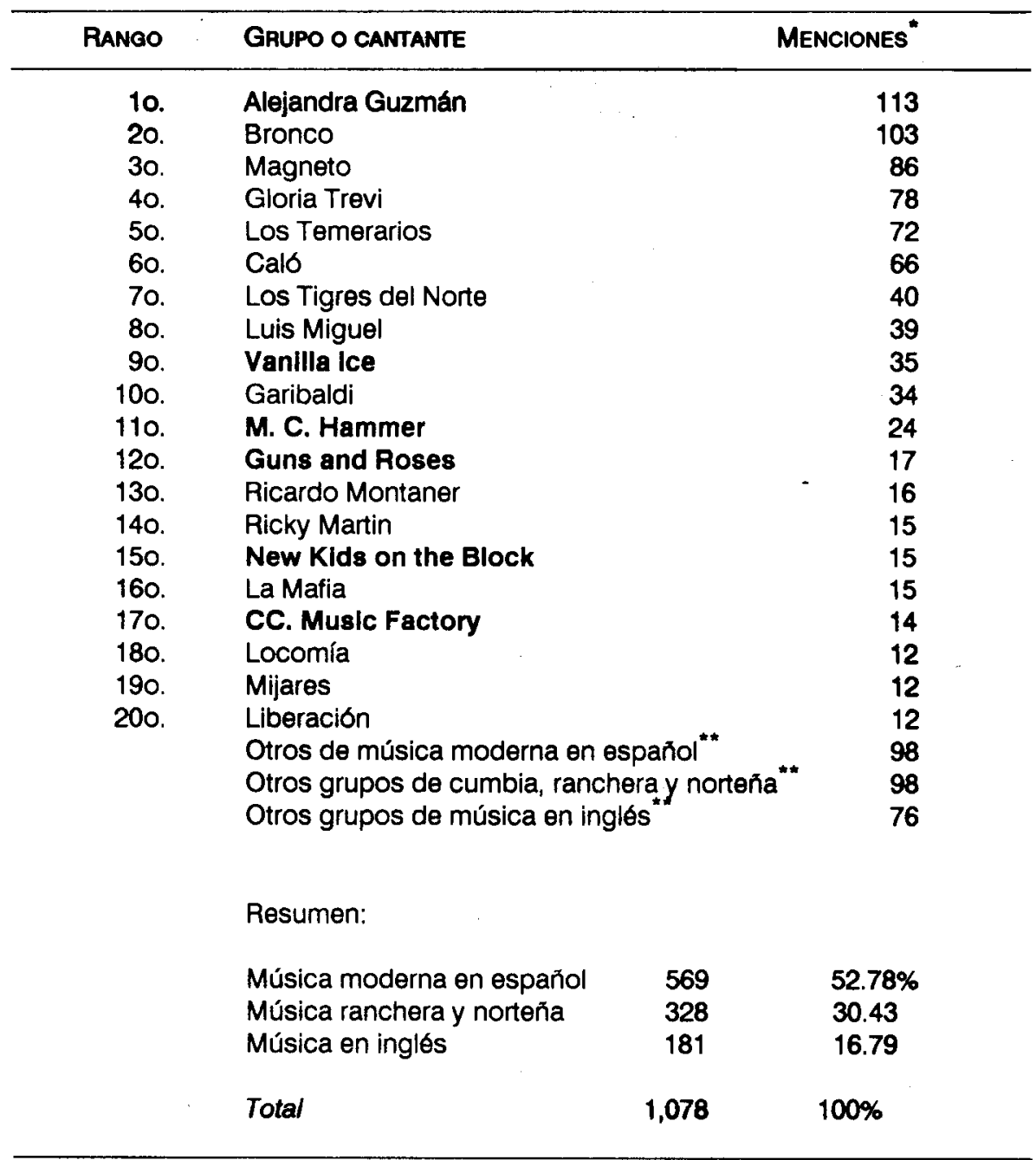

" Cada alumno mencionó dos de sus grupos o cantantes favoritos; el total reportado es el de todas las menciones.

Con menos de doce menciones, cada uno.

el $84.8 \%$ de las cintas vistas por los estudiantes en video y $77 \%$ de las vistas en salas cinematográficas eran de procedencia estadounidense. La gran mayoría de estas 
Cuadro 10. Películas vistas recientemente por los alumnos con videocasetera o en el cine, por procedencia

\begin{tabular}{lcc}
\hline PROCEDENCIA & MENCIONEs* & PORCENTAJE \\
\hline Últimas películas vistas con videocasetera & & \\
& & \\
Películas mexicanas & 91 & 12.69 \\
Películas estadounidenses & 626 & 87.31 \\
TOTAL & 717 & $100 \%$ \\
& & \\
Últimas películas vistas en el cine & & \\
Películas mexicanas & 172 & 19.77 \\
Películas estadounidenses & 698 & 80.23 \\
TOTAL & 717 & $100 \%$ \\
\hline
\end{tabular}

* Cada alumno reportó los nombres de las últimas dos películas vistas por él con la videocasetera o en el cine. Las cintas se clasificaron de acuerdo con su procedencia en "mexicanas" o "estadounidenses". Los totales se refieren al número de títulos mencionados, no al número de alumnos encuestados.

Cuadro 11. Frecuencia con que ven películas mexicanas y estadounidenses con la videocasetera o en el cine

\begin{tabular}{lcc}
\hline Procedencla & MEDIA & DEsv. EsT. \\
\hline Con videocasetera & & \\
Películas mexicanas & $3.02^{*}$ & 1.028 \\
Películas estadounidenses & 3.78 & 1.066 \\
& & \\
En el cine & & \\
Películas mexicanas & 2.69 & 1.207 \\
Películas estadounidenses & 3.14 & 1.363 \\
& & \\
En la televisión & & \\
Películas mexicanas & 3.72 & 0.992 \\
Películas estadounidenses & 3.33 & 1.221 \\
\hline
\end{tabular}

\footnotetext{
"Media en la escala: 1 "Nunca", 2 "Rara vez", 3 "De vez en cuando", 4 "Con frecuencia", 5 "Siempre".
} 
películas se veían dobladas al español o con subtítulos. El hecho de que fueran rentadas en el lado mexicano de la frontera en cadenas nacionales o videoclubes locales o vistas en cines afiliados a cadenas nacionales de distribución reflejan la amplia disponibilidad de filmes estadounidenses en el país entero y no sólo en la región fronteriza. Como muestra de lo anterior se pueden mencionar los hallazgos de González (1992: 257-258) quien reporta que en Comala, Colima, las películas más rentadas en los dos videoclubes existentes eran de procedencia estadounidense.

Ya sea que la exposición a películas estadounidenses sea similar a la que se da en el interior del país o no, el hecho es que los estudiantes de secundaria de esta ciudad fronteriza se exponían a ellas, abriéndose la posibilidad de que se ejerciera algún tipo de influencia ideológica o impacto en su identidad cultural.

\section{Diferencias en la exposición a contenidos estadounidenses por clase social}

Los datos anteriores indican que los jóvenes de secundaria de Nuevo Laredo, como grupo, preferían sus programas televisivos nacionales y la música mexicana sobre sus equivalentes estadounidenses, aunque sin embargo se exponían más a filmes estadounidenses que nacionales. Sin embargo, aún no se ha analizado si en el caso de la exposición a la comunicación de masas en la frontera se da también lo que las investigaciones empíricas previas realizadas en la region han determinado sobre otros fenómenos y procesos culturales: que a mayor clase social, mayor identificación con la cultura estadounidense y mayor distanciamiento de los indicadores relativos a la cultura nacional.

Exposición a programas televisivos estadounidenses. El Cuadro 10 muestra que aunque la clase social no propiciaba diferencias en la frecuencia con que veran programas televisivos mexicanos, sí era relevante para la exposición a los estadounidenses. Los estudiantes de clase alta veían más programas estadounidenses (series como Magnum, Chips y Daktari, dobladas al español) que los de clase media o baja. Esto parece confirmar las afirmaciones de El Colegio de la Frontera Norte: a mayor clase social, mayor predilección por la cultura estadounidense. Hay que aclarar, sin embargo, que los jóvenes de clase alta - aunque proporcionalmente veían más series estadounidenses que los dos grupos restantes - veían significativamente más programas mexicanos que estadounidenses, lo que matiza cualquier 
afirmación simplista sobre el rechazo de esta clase social a los contenidos nacionales.

Exposición a música estadounidense. Con respecto a la frecuencia con que escuchaban música mexicanay estadunidense por clase social, el Cuadro 11 muestra diferencias significativas entre los jóvenes de clase alta y los de clase media y baja. Los primeros oían significativamente más música estadounidense (media $=3.98$ ) que los de clase media (3.13) y baja (2.98). Sin embargo, igual que en el caso de los contenidos televisivos, los estudiantes de clase alta escuchaban más música mexicana (4.12) que estadounidense (3.98), lo que refuerza la conclusión anterior de que si bien tienen mayor predisposición por contenidos estadounidenses, no significa que éstos les resulten más atractivos que los nacionales.

Exposición a películas estadounidenses. Con respecto a las cintas vistas en video, el Cuadro 12 muestra que los jóvenes de clase alta tendían a ver significativamente más filmes estadounidenses (media $=4.09$ ) que los de clase media (3.64) y baja (3.43). En contraste con los contenidos televisivos y musicales, en este caso no se exponían más al cine mexicano que al procedente de Estados Unidos (media $=2.72$ ). Que la recepción de estos contenidos es diferente a la de los anteriores se advierte claramente en los hábitos de exposición de los otros dos grupos. Tanto los de clase media como los de clase baja se exponían más a películas estadounidenses que a las mexicanas, reflejando por primera vez una menor preferencia por los productos nacionales. Esto se explica, en parte, por la mala calidad del cine mexicano, por sus argumentos y por su menor disponibilidad numérica ante la oferta estadounidense en los videoclubes. ${ }^{7}$ Si partimos de la base de que para que se dé una influencia de los contenidos estadounidenses en la identidad cultural de los jóvenes fronterizos, se requiere una exposición intensa a éstos, los del cine en video cumplían con creces esa primera condición.

El panorama de la exposición a películas estadounidenses en salas públicas era un poco diferente. Mientras que los jóvenes de clase altay media seguían prefiriendo ir a ver cintas estadounidenses, los de clase baja preferían las mexicanas (ver Cuadro 12). Esto, en conclusión, señala la importancia de analizar el impacto del cine estadounidense en la identidad cultural de los fronterizos y demuestra la comple-

7 Estas conclusiones están basadas en los comentarios derivados de 30 entrevistas en profundidad con los mismos jóvenes de secundaria que se realizaron como parte del proyectoque aquí se reporta. 
Cuadro 12. Frecuencia con que ven estaciones de televisión mexicanas y estadounidenses

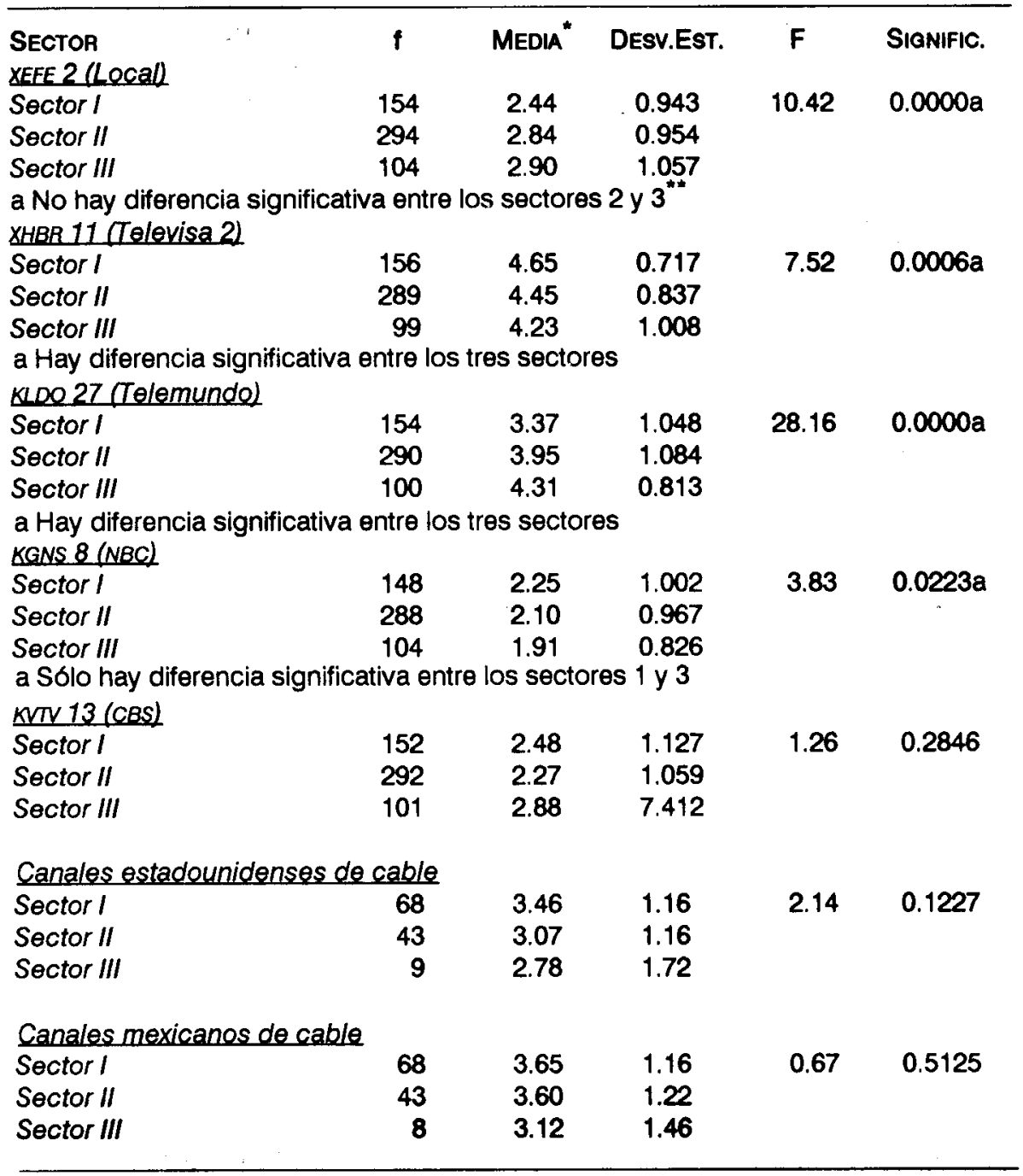

"Media en la escala de: 1 "Nunca", 2 "Rara vez", 3 "De vez en cuando", 4 "Con frecuencia" y 5 "Todos los días".

"* Procedimiento $b$ de Tukey. 
jidad de los procesos de recepción y la coexistencia de diferentes patrones de exposición en los estudiantes de secundaria.

Con respecto a las conclusiones de El Colef, se puede señalar que, en principio, la premisa de que a mayor clase social mayor predilección por la cultura estadounidense (en este caso por la comunicación de masas originada allá) parece suficientemente válida. Los jóvenes de clase alta tendían a exponerse significativamente más a contenidos televisivos, musicales y cinematográficos que sus contrapartes de clase media y baja. Sin embargo, lo anterior no reflejaba necesariamente un desprecio por los medios mexicanos en los estudiantes de clase alta. De hecho, preferían con mucho la televisión y la música mexicanas sobre sus equivalentes estadounidenses. La excepción, pero para todas las clases sociales y quizás en cualquier parte del país, era la exposición a filmes estadounidenses, la cual tendía a prevalecer en detrimento de la exposición al cine nacional.

\section{Conclusiones}

El presente trabajo se planteó cuatro objetivos generales. Los primeros dos buscan ofrecer datos básicos sobre la disponibilidad, acceso y hábitos de exposición a los medios de un segmento específico de la audiencia: 1) conocer la disponibilidad de las diferentes tecnologías de la comunicación masiva (radio, televisión, videocasetera, cable, antena parabólica) entre los jóvenes de Nuevo Laredo, Tamaulipas, ciudad fronteriza con los Estados Unidos; 2) determinar la frecuencia con que se exponían a la radio, la televisión y el cine.

Los dos objetivos restantes abordan algunos aspectos relacionados con los medios y contenidos estadounidenses y la identidad cultural de los jóvenes fronterizos:3) determinar si existe una exposición masiva a los medios de comunicación estadounidenses o a mensajes estadounidenses disponibles en los medios mexicanos por parte de los jóvenes de Nuevo Laredo, y 4) identificar si existen diferencias en la exposición a medios y contenidos nacionales y estadounidenses por clase social, sexo, años de residencia en la frontera y conocimiento del idioma inglés.

La diversidad de hallazgos de esta investigación refleja la complejidad de los procesos de recepción y consumo de medios que se manifiestan entre los jóvenes fronterizos, y valida el enfoque de analizar más integralmente la exposición simultánea a diferentes medios de comunicación evitando el centrarse exclusivamente en la television. 
Respecto del primer objetivo, los hallazgos, resumidos, son los siguientes:

a) La gran mayoría de los alumnos contaban con aparatos de radio y televisión en sus casas;

b) La disponibilidad de videocaseteras, televisión por cable y antenas parabólicas se diferenciaba por nivel socio-económico. A mayor clase social, mayor disponibilidad de estas nuevas tecnologías. De los tres medios, la videocasetera era la más difundida en los tres estratos.

En relación con los hábitos de exposición a los medios, se llegó a los siguientes resultados:

c) Los alumnos veían un promedio de 4 horas diarias la televisión;

d) Escuchaban 2 horas 44 minutos la radio;

e) Veían películas en video casi 3 veces a la semana.

f) Los estudiantes, sin importar clase social, preferían claramente las estaciones televisivas mexicanas o de programación en español sobre las estadounidenses. XEW Canal 2 nacional de Televisa era la que veían con mayor frecuencia;

g) A pesar de contar con más opciones televisivas estadounidenses en el cable, la mayoría de los alumnos con dicho servicio prefería los canales mexicanos; sólo el pequeño grupo de alumnos de clase alta con antena parabólica se exponía con mayor frecuencia a estaciones estadounidenses que a mexicanas.

h) Lo mismo ocurría con la exposición a radiodifusoras. La inmensa mayoría escuchaba estaciones mexicanas con programación en español; las tres radiodifusoras ubicadas en Laredo, Texas quedaban en los últimos cuatro lugares de preferencia.

Respecto de la exposición a contenidos estadounidenses, los hallazgos fueron los siguientes:

i) Los alumnos, sin importar su clase social, preferían con mucho los programas televisivos mexicanos sobre los estadounidenses (incluyendo aquellos doblados al español y disponibles en el canal televisivo local). Catorce de los 16 programas más vistos eran mexicanos (13 de ellos producidos por Televisa); 
los dos restantes (Cara a cara y Ocurrió así) se producían en Miami, pero por Telemundo, en español y con conductores hispanos;

j) Los alumnos preferían la música mexicana sobre la de EU; los de clase alta se inclinaban por la moderna en español, los de baja por la norteña y ranchera.

k) En contraste, los jóvenes se exponían en mucho mayor grado a películas estadounidenses - dobladas al español o con subtítulos - que a las nacionales. Aquí se volvía a presentar el fenómeno ya detectado por El Colef en otros ámbitos culturales: los de mayor clase social tendían a exponerse significativamente más a filmes de Estados Unidos que los de clase baja. Aún así, los de clase media y baja tendían a ver más cintas extranjeras que nacionales, abriéndose la posibilidad de que se ejerciera un impacto en la identidad cultural.

Los resultados discutidos arriba sugieren que, a pesar de la proximidad geográfica con los Estados Unidos y la recepción libre de señales de radio y televisión provenientes de ese país, los jóvenes de Nuevo Laredo comparten los mismos hábitos y preferencias de medios que los receptores del interior de México. En términos de exposición a estaciones televisivas, los jóvenes se inclinaban mayoritariamente por el Canal 2 nacional de Televisa, igual que los segmentos de audiencia que han sido analizados en el D. F., Guadalajara y diversas ciudades del país por Aceves (1991), García Canclini (1993), González (1988) y Montoya y Rebeil (1986). Los suscriptores de cable tendían a preferir, al igual que los de León, Guanajuato (véase Revista Telemundo, 1992), los canales mexicanos. En cuestión radiofónica, los estudiantes neolaredenses preferían en forma abrumadora las estaciones y la música nacionales. Sólamente en el cine se advertía un predominio notorio de mensajes estadounidenses, pero ya se ha sugerido que esto parece repetirse en cualquier parte del país, inclusoen las pequeñas poblaciones, comoComala, Colima (véase González, 1992). Asimismo, los propios estudiantes aclaraban que las películas clásicas del cine mexicano (las de Pedro Infante, Jorge Negrete, Tin Tan y Cantinflas) sí les gustan, no así las recientes, caracterizadas por violencia, pornografía y baja calidad.

Los hallazgos también of recen algunas evidencias sobre los primeros dos niveles requeridos para el estudio científico del impacto de los mensajes estadounidenses en la identidad cultural de las audiencias mexicanas. Como se explicó anteriormente, para que los medios o contenidos estadounidenses ejerzan un impacto 
mente, para que los medios o contenidos estadounidenses ejerzan un impacto determinado en la identidad de los públicos nacionales se requiere el cumplimiento de cuatro condiciones: a) Que haya disponibilidad de medios y contenidos extranjeros en los sistemas de comunicación nacionales, o que se reciba la señal a través de recepción aérea, cable o antena parabólica; b) que las audiencias se expongan a ellos significativamente; c) que la exposición a dichos contenidos redunde en la asimilación de los valores ideológicos extranjeros por parte de las audiencias; y d) que esa asimilación propicie realmente un debilitamiento o pérdida de identidad cultural.

La primera condición, en el caso de Nuevo Laredo y de la frontera norte en general, se cumple con creces. Las audiencias de esta región tienen acceso directo a la señal de distintas estaciones radiofónicas y televisivas, así como a contenidos estadounidenses doblados al español o con subtítulos a través de sus televisoras locales, o la exhibición de películas de esa procedencia en las salas cinematográficas, y renta de las mismas en los numerosos videoclubes.

La segunda condición parecía no cumplirse en el caso de la radio y la televisión, y sólo se cumplía matizadamente en el caso del cine. A pesar de ser uno de los segmentos de edad más propensos a la adopción de manifestaciones culturales extranjeras, los jóvenes neolaredenses preferían abrumadoramente la música en español - tanto la moderna como la popular - y los programas televisivos nacionales. Si la segunda premisa es válida, la poca exposición a contenidos musicales y televisivos estadounidenses sugería que no podía darse por esa vía ni la asimilación de valores ideológicos foráneos ni el posible debilitamiento de la identidad cultural. Caso distinto era el del cine, donde las premisas de disponibilidad y exposición se cumplían cabalmente. Aunque esto abre la vía para que se presenten los dos niveles restantes, queda por determinar en otros estudios si efectivamente la exposición a películas extranjeras redunda en la adopción de manifestaciones culturales ajenas a la idiosincracia nacional, o si dicha exposición se neutraliza por la preferencia simultánea por contenidos televisivos y radiofónicos nacionales.

Por último, en relación con las conclusiones de investigadores de El Colegio de la Frontera Norte de que a mayor clase social mayor preferencia por manifestaciones culturales estadounidenses, este trabajo ofrece evidencias que sugieren su validez para el caso de los medios de comunicación. Como se puede apreciar en la sección de resultados, los jóvenes de clase alta se exponían significativamente en mayor grado a contenidos estadounidenses que sus contrapartes de clase media 
y baja. Esto refuerza los planteamientos previos de estudiar a fondo los procesos de socialización, valores y prácticas culturales de las distintas clases y grupos sociales, así como la apropiación diferenciada de valores y costumbres extranjeras. Cabe reiterar, sin embargo, que si bien los muchachos de clase alta se exponian en mayor grado que sus compañeros a contenidos estadounidenses, éstos tendían a ocupar un nivel secundario respecto de los nacionales, por lo menos en el caso de la música y los programas televisivos.

Todo lo anterior refleja la necesidad de abordar más contextualmente la exposición de los distintos grupos sociales a los diferentes medios de comunicación. De haberse concentrado este trabajo exclusivamente en la televisión, se hubiera llegado a conclusiones muy incompletas sobre la preferencia por contenidos comunicacionales estadounidenses. Aunque más laboriosos, estos estudios de recepción ofrecen una visión más completa sobre los complejos procesos de uso y exposición a los medios que ejercen las audiencias.

Como en la mayoría de las investigaciones, la presente abre más interrogantes de las que contesta. En primer lugar, queda abierta la pregunta de quétan diferentes en términos ideológicos son la televisión y la música comerciales mexicanas de las estadounidenses. Los investigadores críticos que trabajan en la línea de la economía política o del imperialismo cultural aseguran que las diferencias son mínimas. La producción industrial de los mensajes y su tratamiento como mercancías, así como la adopción de tecnologías, géneros y formatos estadounidenses por los medios mexicanos, según estos autores, propicia la semejanza en valores ideológicos y concepciones del mundo. De esta manera, la identidad cultural de los jóvenes de secundaria de Nuevo Laredo no quedaría salvaguardada por su exposición a contenidos nacionales, ya que estos reproducen en gran medida los significados ideológicos capitalistas de sus contrapartes estadounidenses. Un abordaje a esto mismo, por parte de los investigadores culturalistas, sin embargo, llevaría a conclusiones más matizadas. No pueden ser idénticos, por ejemplo, Cándido Pérez o Corazón Salvaje y Alf o Dallas. La polisemia de los mensajes, así comolas diferentes influencias individuales que logran ejercer a nivel micro los profesionales de los medios, sugiere la existencia de valores y significados distintos en los medios mexicanos.

Otras preguntas que deberán responder futuras investigaciones se relacionan con la apropiación específica de los contenidos comunicacionales por parte de los jóvenes fronterizos. ¿Qué procesos de asimilación, rechazo, negociación o refun- 
cionalización se dan en la recepción de los mensajes? ¿De qué manera se integran estos últimos a su vida cotidiana? ¿Qué papel juegan los medios en la conformación de las identidades sociales?

En los próximos años, con la globalización y la intensificación de flujos comunicacionales desde Estados Unidos, Canadá y el resto del mundo, plantearse interrogantes como las anteriores será vital para comprender el impacto de los sistemas de comunicación de masas en la identidad cultural de los mexicanos.

\section{Referencias bibliográficas}

ACEVES Francisco de Jesús (1991): "La televisión y los tapatíos, un atisbamiento al entreveramiento horario de transmisión, menú programático y patrones de exposición". Comunicación y Sociedad Núm. 13 (septiembre-diciembre).

BÉJAR Raúl y Héctor CAPELLO (1988): La conciencia nacional en la frontera norte mexicana. México: UNAM, Centro Regional de Investigaciones Multidisciplinarias.

BUSTAMANTE Jorge (1983): "Tensiones sociales en la frontera norte y la Ciudad de México". Tijuana: Cefnomex (Manuscrito inédito).

(1989): "Frontera México-Estados Unidos; reflexiones para un marco teórico". Frontera Norte Vol. 1, Núm.1 (enero-junio).

(1990): Historia de la Colonia Libertad. Tijuana: Cuadernos COLEF.

CASTELlANOS Alicia y Gilberto LOPEZ (1981): "La influencia norteamericana en la cultura de la frontera norte de México". En Roque GonZÁleZ (comp.) La frontera del norte: integración y desarrollo. México: El Colegio de México.

CEBALlos Manuel (1991): La historia y la epopeya en los origenes de Nuevo Laredo. Cuadernos de la Facultad Núm. 5. Nuevo Laredo, Tamaulipas: Universidad Autónoma de Tamaulipas. 
(1992): "La epopeya de la fundación de Nuevo Laredo: el nexo entre la tradición y la historia". En Manuel VALENZuel.A (comp.) Entre la magia y la historia. México: Programa Cultural de las Fronteras/El Colegio de la Frontera Norte.

CREMOUX Raúl (1968): La televisión y el alumno de secundaria del Distrito Federal. México: Centro de Estudios Educativos.

DE CERTEAU Michel (1987): "La invención de lo cotidiano". Espacios Vol. 4, Núm. 11.

DE LA GARDE Roger (1987): "Is there a market for foreign cultures?". Media, Culture \& Society Vol. 9, Núm. 2.

ESPINOZA VAlle Víctor (1990): Don Crispin: una crónica fronteriza. Tijuana: El Colegio de la Frontera Norte.

ESTEINOU Javier (1991-92): "Los medios de comunicación frente al TLC". Cultura Norte Año 4, Núm. 5.

Fernández Collado Carlos, Pilar Baptista y Débora Elkes (1984): La televisión y el niño. México: Nueva Biblioteca Pedagógica.

FUENTES Raúl y Enrique SÁNCHEZ RUIZ (1992): "La investigación de la comunicación en México, los retos de la institucionalización". En Cuadernos de Comunicación y Prácticas Sociales Núm. 3, Universidad Iberoamericana.

FUENZALIDA Valerio (1989): Visiones y ambiciones del televidente. Chile: Ceneca.

GARCía CANCLINI Néstor (1988): "Cultura transnacional y culturas populares". En N. GARCÍA CANCLINI y Rafael RonCAGLIOLO (eds.) Cultura transnacional y culturas populares. Lima: IPAL.

(1990): Culturas hibridas. México: Conaculta/Grijalbo. 
(1991): "El consumo sirve para pensar". Diá-Logos de la Comunicación Núm. 30. Lima: FELAFACS.

(1992): "Los estudios sobre comunicación y consumo: el trabajo interdisciplinario en tiempos neoconservadores”. Diá-Logos de la Comunicación Núm. 32. Lima: FELAFACS.

- (ed.) (1993): El consumo cultural en México. México: CONACULTA.

- y Patricia SAfa (1989): Tijuana: la Casa de Toda la Gente. México: INAH-ENAH, Programa Cultural de las Fronteras.

GONZÁLEZ Jorge (1988): "La cofradía de las emociones interminables". Estudios sobre las Culturas Contemporáneas Vol. 2, Núms. 4-5. Colima: Universidad de Colima, Programa Cultura.

(1992): "Video tecnología y modernidad; por los dominios de Pedro Páramo". En Javier EsTEINOU (ed.) Comunicación y democracia: VI Encuentro Nacional CONEICC. México: CONEICC.

Intermedios (1992): "Hábitos de lectura de periódicos en el Valle de México", Núm. 4 (octubre).

Intermedios (1992): "Hábitos de consumo televisivo en el Valle de México", Núm. 3 (agosto).

Lozano José Carlos (1990): "Identidad cultural, actitudes políticas y valores socioculturales en Tijuana" Estudios Fronterizos Núm. 22 (mayo-agosto).

- (1991a): Prensa, radiodifusión e identidad cultural en la frontera norte. Tijuana: Cuadernos COLEF.

(1991b): "Enfoques teóricos para el estudio de la cultura en la frontera de México con los Estados Unidos”. Río Bravo, a bilingual joumal of international studies Vol. 1, Núm. 1 (otoño). 
(1992): “Apropiación de tradiciones anglosajonas en la frontera norte: el caso del Día de la Coneja". En Manuel VAlenzuela (comp.) Entre la magia y la historia. México: Programa Cultural de las Fronteras/El Colegio de la Frontera Norte.

LULL James (1990): Inside family viewing. Londres: Routledge.

MALAGAMBA Amelia (1986): La televisión y su impacto en la población infantil de Tijuana. Tijuana: CEFNOMEX.

MARTÍN BARBERo Jesús M. (1988b): Procesos de comunicación y matrices de cultura. México: FELAFACs/Gustavo Gili.

y Sonia MUÑoz (coords.) (1992): Televisión y melodrama. Bogotá: Tercer Mundo Editores.

MONSIVÁIS Carlos(1981): "La cultura de la frontera". Estudios fronterizos. México: ANUIES.

(1983): "Interacción cultural fronteriza". En Mario MiRANDA y James WILKIE (eds.) Reglas del juego y juego sin reglas en la vida fronteriza. México: ANUIES/PROFMEX.

Montoya Alberto y María A. ReBell (1986): "Commercial television as an educational and political institution: a case study of its impact on the students of Telesecundaria". En Rita ATwOoD y Emile MCANANY (eds.) Communication and Latin American society: trends in critical research, 1960-1985. Madison, Estados Unidos: The University of Wisconsin Press.

Murrieta Mayo y Alberto Hernández (1991): Puente México: la vecindad de Tijuana con California. Tijuana: El Colegio de la Frontera Norte.

PRIETODaniel (1984): “Sobre la teoría y el teoricismo en comunicación”. En Fátima FERNÁNDEZ y Margarita YePES (comps.) Comunicación y teoría social. México: UNAM. 
Revista Telemundo (1992): "¿Qué canal prefieren ver los espectadores de TV-Cable?". Núm. 4.

RIDING Alan (1986): Distant neighbors: a portrait of the Mexicans. Nueva York: Vintage Books.

Ross Stanley (1979): "Introduction". En Stanley ROSS (ed.) Views across the border. Albuquerque: University of New Mexico Press.

SALAS PORRAS Alejandra (1989): "La frontera: una larga lucha por la independencia". En Alejandra SALAS PORRAS (coord.) Nuestra frontera norte: ("tan cerca de los $E U^{\prime \prime)}$. México: Editorial Nuestro Tiempo.

SÁNCHEZ RUIZ Enrique (1989): "Televisión y representaciones políticas de escolares tapatíos". Comunicación y Sociedad Núm. 7.

- (1993): "La investigación de la comunicación en tiempos neoliberales". Revista Mexicana de Comunicación Año 5, Núm. 27 (enero-febrero).

TOUSSAINT Florence (1990): Recuento de medios fronterizos. México: Fundación Manuel Buendía/Programa Cultural de las Fronteras.

Valenzuela Manuel (1988): iA la brava ése!: Cholos, Punks y Chavos Banda. Tijuana: El Colegio de la Frontera Norte.

- (1993): “Ambitos de interacción y consumo cultural en los jóvenes”. En Néstor García CANClini (coord.) El consumo cultural en México. México: Conaculta.

ZÚNIGGA Víctor (1991): "Los locos del barrio o la ostentación del estigma social y fronterizo en una pandilla de Matamoros, Tamaulipas". Rio Bravo Vol. 1, Núm. 1 (otoño). 